\title{
Medical workforce review using the RCP guidance on safe medical staffing demonstrating wide variation of workload to workforce ratio between medical teams within a single
} trust

\author{
Authors: Thomas Weetman, Deanne Bell, Gareth Watts* and Fang En Sin*
}

\section{Introduction}

The Royal College of Physicians (RCP) published guidance on safe medical staffing in 2018. ${ }^{1}$ Its recommendations were derived by estimating the workload generated by each clinical activity.

In our trust, there is a perception of a high junior doctor workload and an imbalance of workload between teams, which can compromise patient safety, work efficiency and training quality. Acting on this, we aim to:

> compare the workload per tier 1 (pre-registrar) doctor between medical teams

> compare the trust's tier 1 staffing level against the RCP benchmark, focusing on staffing of medical wards in-hours.

\section{Methods}

Twenty medical teams across Brighton and Sussex University Healthcare Trust (encompassing two hospitals) were assessed.

The clinical activity of each team (number of new and preexisting inpatients, and discharges) was collected over a 2-week period using inpatient lists and electronic data. Workload was calculated as 'average hours of work required per week per team' based on estimations in the RCP report.

In calculating the average hours of ward work contributed by each tier 1 doctor per week, on-call commitments were accounted for, in addition to leave entitlements.

With this data, we then:

> determined the ratio of workload to workforce per team, and compared between teams

> compared the number of doctors needed as per the RCP guidance with actual staffing levels.

To help validate the guidance, we assessed additional hours worked using exception reports and diary cards from tier 1 doctors for 1 week.

Authors: Brighton and Sussex University Hospitals NHS Trust ${ }^{*} \mathrm{RCP}$ chief registrar

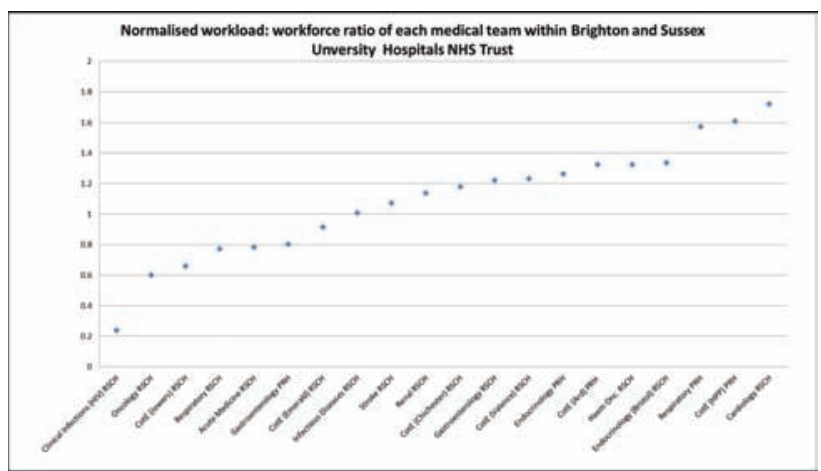

Fig 1: Variation in normalised workload to workforce ratio between medical teams within the trust, with 1.0 being the median

\section{Results}

The average number of inpatients per team per day was 23 (range $3-51)$, with four new patients (0-13) and two discharges (0-6). The average hours of weekly ward work per team was 55.6 (6.4-88.3), with 5.4 doctors per team, each contributing 28.8 hours of ward presence a week.

The normalised workload to workforce ratio demonstrated a wide variation, ranging from 0.24 to 1.7 (with 1.0 representing the median; Fig 1). The average workload to workforce ratio was higher at PRH (1.3) than RSCH (1.0).

Overall, there was more than the recommended number of doctors (average actual:recommended ratio 1.9). Despite meeting the minimum staffing requirement of the RCP, staff are routinely working beyond contracted hours. A total of 77 hours were exception reported in 1 month, and the 'diary card' reported a total of 30.4 hours of extra work in 1 week.

Possible explanations include: staff and system inefficiencies; mismatch of workload to staffing level due to variations in workload and rota management; high workload from high acuity and complex patients. In addition, the hours of ward presence did not account for staff breaks and mandatory teaching. 


\section{Conclusion}

The RCP guidance forms a basis for trusts to benchmark their workforce. We demonstrated the importance of regular reviews of medical staff distribution, in order to ensure safe, efficient delivery of care. The guidance stipulates a minimally safe staffing level; however the reality of maintaining a progressive care system may necessitate more doctors. The RCP recognises that the report is 'the start of an ongoing process', and we hope that our findings contribute to this process.

\section{Reference}

1 Royal College of Physicians. Guidance on safe medical staffing. Report of a working party. London: RCP, 2018. Available at: www. rcplondon.ac.uk/projects/outputs/safe-medical-staffing [Accessed 28 February 2019]. 